\title{
THE DYNAMICS OF THE SIERPIŃSKI CURVE
}

\author{
JAN M. AARTS AND LEX G. OVERSTEEGEN
}

(Communicated by James E. West)

\begin{abstract}
The Sierpinski curve $X$ admits a homeomorphism with a dense orbit. However, $X$ is not minimal and does not admit an expansive homeomorphism.
\end{abstract}

\section{Statement of the theorem}

A Sierpinski curve is a subset of the 2-sphere $S$ that remains after removing from $S$ the interiors of a null sequence of mutually disjoint closed disks whose union is dense in $S$.

This construction of the Sierpinski curve $X$ is in fact a characterization of it; the Sierpiński curve thus constructed is unique [15]. For basic definitions we refer the reader to [8]. During the 1992 Spring Topology Conference several questions regarding the dynamics of the Sierpinski curve were raised. We note that, as is well known, the Sierpinski curve does not admit a nontrivial flow. The following theorem gives a solution to these problems.

Theorem 1. Let $X$ be the Sierpinski curve. Then:

(1) $X$ admits a transitive homeomorphism, but

(2) $X$ is not minimal, and

(3) $X$ does not admit an expansive homeomorphism.

Statement (2) answers a question by Gottschalk [7]. Fact (3) has already been observed by Kato [11]. The short proof provided here is in the same spirit as the proof of (2). Other interesting results regarding expansive homeomorphisms on curves can be found in [10]. The statements will be proved in the order (2), (3), (1).

Proof of Theorem 1. (2) Suppose $f: X \rightarrow X$ is a homeomorphism. We shall prove that $f$ is not minimal by exhibiting a nonempty proper closed subset of $X$ that is invariant under $f$. Note that $X$ has two types of circles, namely, separating and nonseparating ones. The nonseparating circles are the boundaries of the removed disks. The union of the nonseparating circles is a (nonclosed)

Received by the editors April 28, 1992.

1991 Mathematics Subject Classification. Primary 54H20; Secondary 34C35, 58F13.

Key words and phrases. Chaos, minimal, transitive, dense orbit, expansive. 
invariant subset. Since $f$ permutes the boundaries of the complementary domains of $X$ in $S$, we can extend $f$ to a homeomorphism $F: S \rightarrow S$. Now, consider the decomposition $\mathscr{D}$ of $S$ whose nondegenerate elements are the closures of the complementary domains. Since the nondegenerate elements form a null sequence, the decomposition $\mathscr{D}$ is upper semicontinuous [14]. The resulting quotient space $Y$ is homeomorphic with the 2-sphere $S$ [12]. Let us denote by $\tilde{f}$ the homeomorphism of $Y$ onto itself that is induced by $F$. From the Lefschetz fixed-point theorem [6, Theorem XVI, 3.4], the map $\tilde{f}$ has a periodic point. In particular, $Y$ has a nonempty proper closed subset that is invariant under $\tilde{f}$. It follows that $F$, and also $f$, is not minimal. This completes the proof of (2).

(3) Suppose $f: X \rightarrow X$ is an expansive homeomorphism. Since no circle admits an expansive homeomorphism [9], no nonseparating circle is invariant under a power of $f$. Hence, for a given nonseparating circle $C$, $f^{n}(C) \cap f^{m}(C)=\varnothing$ for all integers $n \neq m$. Since there are only finitely many "large" nonseparating circles, for any $\varepsilon>0$, there exists an $N$ such that $\operatorname{diam}\left(f^{n}(C)\right)<\varepsilon$ for $|n|>N$. It follows that $f$ cannot be expansive.

(1) Besicovitch [2] has shown that there exists a homeomorphism of the open annulus with a dense orbit which preserves the radial lines. Hence it extends over the closed annulus $A$. The annulus can be seen as a subset of $S$ obtained by removing two disks. Our goal is to insert a null sequence of mutually disjoint closed disks whose union is dense in $A$. After removing the interiors of the inserted disks we end up with the Sierpinski curve together with the required homeomorphism. Let $f: A \rightarrow A$ be a homeomorphism as above, and let $x$ be a point such that the orbit $\left\{f^{n}(x)\right\}_{n \in Z}$ is dense in $A$. Put $x_{n}=f^{n}(x)$ for $n \in \mathbf{Z}$.

We shall construct an inverse sequence $X_{n}$ of copies of $S$. We think of $X_{0}$ and $X_{1}$ as $S$ with the annulus $A$ as a closed subset. Let $\mathscr{F}_{0}$ be a foliation consisting of radial line segments emanating from the point $x_{0}$. Suppose $X_{n}$ has been constructed. Assume first that $n$ is even and greater than 0 . We will insert a disk at the point $x_{n / 2}$. Write $n / 2=m$. In case $n$ is odd a similar construction inserts a disk at the point $x_{-(n+1) / 2}$.

Let $\mathscr{F}_{m}$ be the foliation emanating from $x_{m}$ that is obtained by transporting the foliation $\mathscr{F}_{0}$ by $f^{m}$. Hence each leaf of $\mathscr{F}_{m}$ is a half-open arc which can be labeled with a real number $\alpha$ between 0 and $2 \pi$. Let $X_{n+1}$ be the space obtained from the disjoint union of $X_{n} \backslash\left\{x_{m}\right\}$ and a disk $D_{m}$ by compactifying the leaf with number $\alpha$ with the point with argument $\alpha$ on the boundary of $D_{m}$ for each $\alpha \in[0,2 \pi)$. Let $q_{n}: X_{n+1} \rightarrow X_{n}$ be the natural quotient map which identifies $D_{m}$ to the point $x_{m}$. Let

$$
X_{\infty}=\lim _{\leftarrow}\left(X_{n}, q_{n}\right),
$$

and let $\tilde{q}$ be the natural projection $X_{\infty} \rightarrow X_{0}$. Note that the decomposition $\left\{\tilde{q}^{-1}(x) \mid x \in S\right\}$ has only countably many nondegenerate elements which form a null sequence of disks. For convenience we will denote the nondegenerate elements of the decomposition also by $D_{m}$. It can be seen that this decomposition is shrinkable, and hence $\tilde{q}$ is a near homeomorphism [4, p. 23]. Hence, $X_{\infty}$ is homeomorphic with $S$. 
Observe that the continuum obtained by deleting the interiors of the inserted disks $D_{m}$ from $\tilde{q}^{-1}(A)$ is a Sierpinski curve $X$. The required map $\hat{f}$ on $X$ will be induced by the map $f$ as follows. Note that the map $\hat{f}$ can be defined in a natural manner away from the null sequence $\left\{D_{m}\right\}$. Each point with argument $\alpha$ in the boundary of $D_{m}$ is in the closure of a unique leaf $L_{\alpha}$ of $\mathscr{F}_{m}$. Let $\hat{f}(\alpha)$ be the unique point in the boundary of the disc $D_{m+1}$ which is in the closure of the leaf $\hat{f}\left(L_{\alpha} \backslash \bigcup_{n \in \mathbf{Z}} D_{n}\right)$. This defines the required homeomorphism $\hat{f}$.

Remark 1. It seems likely that results by Oxtoby and Ulam [13] can be modified to obtain a homeomorphism of the 2-sphere $S$ with a dense orbit and a dense set of periodic points. In fact, the existence of such a homeomorphism was announced by Beverly Brechner at the Joint Summer Research Conference on Continuum Theory and Dynamical Systems held at Humboldt State University in 1989. Using this homeomorphism in the construction above yields a transitive iomeomorphism of the Sierpinski curve with a dense set of periodic points. By a recent result of Banks et al. [1], such a homeomorphism must have sensitive dependence on initial conditions and, hence, is chaotic in the sense of Devaney [5]. Using Cannon's characterization of the higher-dimensional Sierpinski curves [3], appropriate generalizations to higher dimensions are also possible.

We end with

Problem 1. Do the results of Theorem 1 and Remark 1 also hold for the Menger curve?

\section{REFERENCES}

1. J. Banks, J. Brooks, G. Cairns, G. Davis, and P. Stacey, On Devaney's definition of chaos, Amer. Math. Monthly 99 (1992), 332-334.

2. A. S. Besicovitch, A problem on topological transformations of the plane, Fund. Math. 28 (1937), 61-65.

3. J. W. Cannon, A positional characterization of the $(n-1)$-dimensional Sierpinski curve in $S^{n} \quad(n \neq 4)$, Fund. Math. 79 (1973), 107-112.

4. Robert J. Daverman, Decompositions of manifolds, Academic Press, New York, 1986.

5. R. L. Devaney, An introduction to chaotic dynamical systems, Benjamin-Cummings, Menlo Park, CA, 1986.

6. James Dugundji, Topology, Allyn and Bacon, Boston, MA, 1966.

7. W. H. Gottschalk, Minimal sets: an introduction to topological dynamics, Bull. Amer. Math. Soc. 64 (1958), 336-351.

8. W. H. Gottschalk and G. A. Hedlund, Topological dynamics, Amer. Math. Soc. Colloq. Publ., vol. 36, Amer. Math. Soc., Providence, RI, 1955.

9. J. F. Jakobson and W. R. Utz, The nonexistence of expansive homeomorphisms of a closed 2-cell, Pacific J. Math. 10 (1960), 1319-1321.

10. Hisiao Kato, Expansive homeomorphisms in continuum theory, Technical Report 13 (1991), Faculty of Integrated Arts and Sciences, Hiroshima Univ., Hiroshima 730, Japan.

11. The nonexistence of expansive homeomorphisms of peano continua in the plane, Topology Appl. 34 (1990), 161-165.

12. R. L. Moore, Concerning upper semicontinuous collections of compacta, Trans. Amer. Math. Soc. 27 (1925), 416-428. 
13. J. C. Oxtoby and S. M. Ulam, Measure preserving homeomorphisms and metrical transitivity, Ann. of Math. 42 (1941), 874-920.

14. G. T. Whyburn, Analytic topology, Amer. Math. Soc. Colloq. Publ., vol. 28, Amer. Math. Soc., Providence, RI, 1942.

15. $\longrightarrow$, Topological characterization of the Sierpiniski curve, Fund. Math. 45 (1958), 320324.

TUdelft, Faculteit Wiskunde en Informatica, Postbus 356, 2600 AJ Delft, The NETHERLANDS

E-mail address: aartsetri.tudelft.nl

University of Alabama at Birmingham, Department of Mathematics, Birmingham, AlABAMA 35294

E-mail address: oversteecmath.uab.edu 